\title{
Assessment and coping strategies for stress and depression among the terminally ill patients: Focus on cancer
}

\author{
Suantak Demkhosei Vaiphei \\ ${ }^{1}$ Department of Psychology, Assam Down Town University, Guwahati, India
}

Received: 2021-08-25.

Accepted: 2021-11-18

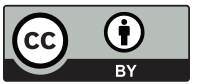

This work is licensed under a Creative Commons Attribution 4.0 International License

J Clin Med Kaz 2021; 18(6):15-19

Corresponding author:

Suantak Demkhosei Vaiphei.

E-mail: gdsuantak@yahoo.com;

ORCID: 0000-0002-4134-2926

\begin{abstract}
The concept of psychological depressive symptoms affecting the terminally ill patient still stood as an unsolved mystery in many clinical practices. The psychological traumatic and depressive stressors are the factors responsible for destroying the quality of life, and affecting the terminally ill individual in the most degrading ways. Alongside the physical pain symptoms, the terminal ill experience is always accompanied by several unwanted mental disharmonies and emotional sufferings like toxins, mood disorder, trauma, and low self-esteem. The higher level of stress and depression determines the growth of tumors and cancer metastasis rate in any terminal diagnosis. This makes the assessment of the psychological non-pain symptoms essential alongside the physical pain symptoms. Failing to acknowledge the patient's traumatic experience, and depressive stressors will make the treatment unproductive and increase suicidal activities.
\end{abstract} cancer

Key words: stress, depression, stressors, terminal diagnosis,

\section{Introduction}

Terminal illness in its nature of existence is a traumatic event with stressful experience, resulting in developing the highest psychological depressive symptoms. Unlike any other ill diagnosis, the psychological depressive stressors in terminal ill experience worsen the individual physical pain symptom and increase tumor growth rate into its advanced stages in the most fruitful ways. It is essential to understand the tumor types to deal effectively with the patient's stressful and depressive symptoms in clinical practices. Several research findings suggested that it is not the tumor or pain symptoms alone that destroyed the patient hope and wellbeing. It is instead the depressive stressors that completely disturb the unique peaceful environment. Thus, understanding the psychological non-pain symptoms alongside the physical pain symptom treatment will better understand the patient's cognitive process with the stressors and its behavior outcomes in any terminal diagnosis [1]. The psychological assessment interventions in the patient's traumatic and depressive experience are also visible in delivering quality of life, controlling the growth of cancer metastasis rate, and enhancing the patient surviving rate.

Purpose: To study the effect of the psychological stressor on various parts of immunological function and associated with cancer. The aim is also to bring out some of the essential assessment methods and propose strategies in dealing with stress and depressive symptoms.

Material and methods: The proposed study is an analytical method basing on the existing documents and literature.

\section{Results and discussion}

Cancer is a disease that affects individual physical health, emotional feeling, social life, and having several psychological disorders as its outcomes. Being with cancer is when the individual develops unwanted habits like excessive use of alcohol and other related drugs to eased down their emotional suffering, pain symptoms, and mental disharmony. It is the times when they need people's support the most to deal with their isolation, 
loneliness, hopelessness, anxiety, and other depressive stressors. Psychological depressive stressors are found to be the most neglected area of care and are sometimes considered symptoms not treated in clinical practices. This negligence hugely affected the individual physical health stability and a higher level of disability. The story of depression is higher in women with terminal illnesses than their male counterparts. The psychological approach to terminal care with family and loved ones supporting the patient to face the existential challenges is visible as the core coping mechanism that delivers the quality of life and wellbeing in the end-of-life care.

\section{Stress and depression in terminal ill experience}

Though the advanced medical science technologies effectively address the wide-ranging needs of terminally ill patients, it mostly confined to the patient physical pain symptoms alone. Terminal ill diagnosis, unlike other illnesses, requires a whole-person treatment that acknowledges the psycho-emotional sufferings that affected the patients in the most degrading ways. Terminal illness is accompanied by several unwanted experiences that hugely disturb the individual wellbeing and quality of life. Some of the stressors that pre-occupied the terminally ill patients are trauma, anxiety, loss of will to live, and other mental disharmonies that serve as the underlying threat to patient quality of life and wellbeing [1,2]. Among all the factors, death anxiety is the main contributing factor affecting the patient immune system, unhealthy relationships, loss of sense over selfesteem, and poor decision making towards treatment plan and policy. The psychological stress and depression associated with the initial stage of cancer and its metastasis hugely decrease the patient's quality of life and wellbeing. Stress in its nature of existence is an adaptive reaction in cancer metastasis that usually produces physical, mental, and behavioral changes in patient terminal experience. It is a non-specific psychological reaction towards life situations' external and internal demands beyond the average human ability to deal with, killing the brain cells. Comparatively, stress is visible as the most common experience among terminally ill patients with long-term psychological distress [2]. In one of the latest findings, depression and stress are the two everyday unwanted experiences among cancer patients in India $(n=320 ; 72.5 \%)$ [3]. Depression is higher in the age group of 18-40 among the women with breast cancer patients in India ( $\mathrm{n}=270 ; 96.7 \%)$ [4]. Depression, on the other hand, is more than just a feeling of worthlessness or mood disorders; it is a symptom that is linked with human brain chemicals of serotonin and norepinephrine, which causes several pains like joint and back pain with sleep disorder and can even lead to a depressive episode. In the World Health Organization (WHO) latest report, depression accounts for patients adjusted with a physical disability, mental and behavior disorder to the patient of all ages [5].

Depression and stress are the two most psychological disorders among terminally ill patients in India. It decreases the patient's health stability, heightened self-depreciation, reduces energy, and is different from grief. The underlying features of depression that terminally ill patients usually experience include bipolar disorder, clinical depressive disorder, persistent depressive disorder, and seasonal affective disorder. The degree of depression varies from the individual experiences, and it has no age bar. The recent findings in India concluded that depression is higher among terminally ill women patients than the men group [6]. In another latest study among cancer patients in India, the depression rate ranges from $4.4 \%$ to
$89.9 \%$, emphasizing emotional distress. The rate of depression increases as per the stage of the cancer metastasis. On the other hand, lack of awareness, ignorance, social stigma, and discrimination becomes the underlying factors for worsening the patient's mental health conditions. Depression has no age bar and is hugely responsible for suicidal activities, which India is considered the most depressed country in the world. Some of the most depressive symptoms are loss of appetite, loss of selfesteem, energy, concentration, and slow cognitive process [7].

The genetic and biological factor plays an essential role in a patient experiencing depression and stress. Specific genes increase the risk of developing a mental illness, depending on one's life situation, which may trigger it like abuse or trauma (life experiences). While some mental health issue arises through the parent environmental exposures resulted in the child mental disorder. Exposure to environmental stressors, inflammatory conditions, toxins, alcohol, drugs while in the womb can sometimes make the child suffering from mental illness. Brain chemistry is also another cause for mental health problems; when the neurotransmitters that carry signals from one part to the other parts of the brain and body are impaired, nerve receptors and nerve systems change dramatically, leading to depression and other emotional disorders [8]. Cancer/terminal illness is a unique experience with both the disease and the situational challenges that produce uncertainty over life and huge psychological effects that disturb the individual nature of existence in the most rigorous way. It is essential to acknowledge the adaptive patient capacity over their environmental challenges and understand how the individual responds to the stressors events in terminal experience. Knowing the level of patient sense of sensitivity towards the stressors event is also essential in developing the coping strategies in the most effective way. Looking at the current condition of India, the depressive stressors that put the individual life into miserable conditions and severe problems in daily life and relationships remain unconcerned. Moreover, the signs and symptoms of depression can vary depending on the individual's mental or psychological state. In general mental health symptoms can affects emotions, behaviors, and the cognitive process [9].

\section{Assessment}

Psychological stress, anxiety, and depression are the most common adverse outcomes in any terminal experience, which require special consideration in attending to the patient emotional needs and mental problems through effective coping strategies in the clinical practices. Coping is an ongoing process that needs several cognitive efforts and energy to deal with the depressive stressors; it requires skills and techniques to give the terminally ill patient the ability to adjust and overcomes the environmental challenges or reduce the stressors events in life. Though the level of depression and stress differ in terminally ill men and women, the coping strategies don't differ, in which the two most effective coping mechanisms are social support and problem-solving techniques. In dealing with depressive stressors, studying the patient's past and present history is essential for practical problem solving, decoding, and addressing the stressors in the most effective and appropriate ways. The hypothalamic-pituitary-adrenocortical axis (HPA) and the sympathetic-adrenal-medullary (SAM) system of the human organs responsible for mood disorders and negative emotional feelings demand special assessment in palliative end-of-life care $[10,8]$.

The HPA and SAM not only influence the effects of stress in cancer metastasis but also have several disease risks related 
to physiological processes, affecting immune cells' activity, including natural killer (NK) cells, T cells, and macrophages as the outcomes of activating the stressful stimuli's. In dealing with psychological stress, the involvement of the family and friends to give emotional support to the patient is considered to be the best coping mechanism to fight against the immunological and endocrinological consequences of the patient psychological functioning. It is also visible that psychosocial-emotional supports is an effective mechanism in reducing tumor metastasis and associated symptoms. It serves as the factor that controls the degree of anxiety and depressive stressors that regularize the individual peripheral and central nervous system functioning in the most effective ways. Immuno-modulatory therapy is also an essential assessment in dealing with patient depressive stressors symptoms in clinical practices [11,9]. The psychotherapeutic intervention is more effective than psychotropic medicines with regards to patient recovery from depression and wellbeing. Moreover, the mere use of counseling therapy alone without psychotherapy has minimal impact on dealing with the depressed situation of the terminal patient. Person-centered therapy with a holistic approach to terminal care can successfully encounter psychological distress stems, emotional needs, reconstruct mental stability, and build practical coping skills. The early intervention of psycho-stimulant substances like dextroamphetamine or methylphenidate is also a well-recognized therapy against insomnia and hugely improves patient mood and wellbeing [12].

The intervention of the psychological approach to terminal care has been visible effective in three essential dimensions for patient quality of life; in effectively reducing the psychological depressive stressors, increasing the patient survivor period, and decreasing the cancer metastasis rate. The collaborations of psychotherapy and psychopharmacological approach to terminal care are also effective in reducing the rate of tumor growth and boosting the functions of the immune system [13]. The followings are the standard existing measuring scales to effectively measures the level of stress and depression in terminal ill diagnosis.

Figure 1 - Stress Measuring Scales [12,13]
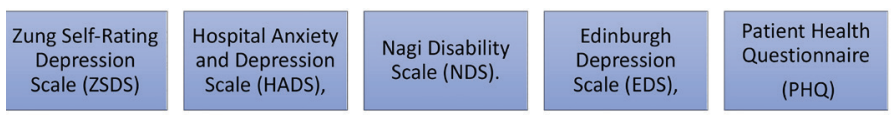

The Hospital Anxiety and Depression Scale (HADS) is a fourteen-item scale depression rating tool use to indicate the antidepressant medical response, while Zung Self-Rating Depression Scale (ZSDS) is a twenty-item self-reporting depression measurement scale use to show the presence or absence or the level of depressive symptoms in palliative endof-life care patients. The Edinburgh Depression Scale (EDS) is a ten-item postnatal depression tool used to measure the symptoms like guilt, hopelessness, mood swing, and another related cognitive process in a terminally ill patient. The Nagi Disability Scale (NDS) is the clinical instrument that identifies the degree of psycho-emotional suffering and other associated issues among cancer survivors. The Patient Health Questionnaire (PHQ-2) is used in screening cancer patients for undiagnosed depression. It is effective in screening larger groups of patients for depression $[14,8]$.

The use of verbal screening instruments by forming some relevant questionnaires regarding the low and high mood of a patient undergoing depression can also enhance the treatment policy to address the depressive stressors. According to DSM-
IV (Diagnostic and Statistical Manual of Mental Disorders), depression is mainly associated with patient loss of interest in daily activities, difficulty in making a quality decision, psychomotor agitation, and suicidal motive because of the disease effects, in which the psychological symptoms of depression is seen as the main characteristic of its outcomes. Thus, the early initiation of the psychological treatment within an adequate period needs to be assessed. If the depressive symptom of hopelessness is not addressed in its early period, it can easily combine with the grief response that usually causes the loss of specific abilities in the patient's terminal experience. The primary concern of the clinician should be adequate control over pain and symptom management and other disease risks in advanced patient illness and to prevent the patient from certain depressive disorders through any possible means [15,13]. Most importantly, the assessment should be regularly, including the appropriate time and place, intensity, quality of evaluation. It is also essential for the clinician working in palliative end-of-life care to identify the difference between physiological tolerance, physical and psychological dependency, and pseudo-addiction in assessing the patient [16].

\section{Suggested coping strategies}

Among all the effective coping mechanisms against stress and depression in terminal ill experiences, drawing a positive attitude to self and the given environmental challenges by accepting the life situations beyond control and adjusting is the most effective coping mechanism for those with terminal ill experience. Striving towards self-esteem and learning to relax over sensitive issues, rather than being aggressive towards what life brings. Adopting the habit of regular physical exercise, managing a balanced diet, avoiding things that create sleeping disorders, and abstaining from the excessive use of alcohol and other related drugs are the core factors to eliminate stress and depression and maximize the quality of life by minimizing the depressive stressors. Diagnosis with cancer/terminal illness is a challenging experience filled with anxiety, negative emotional feelings, and mental disharmony, yet understanding the kind of tumor involves, its metastasis, treatment policy, and the side effects would help in creating appropriate coping skills and the ability to deal with it in the most un-harmful way. Knowing the cultural and religious background is essential for every clinician, as in some cultures, death and dying are taboo that has no place for public discussion [16,17]. Preserving the patient autonomy by respecting the choice of the patient with regards to the disease risks information and the inclusion of the patient in the decisionmaking management team is also essential to produce a quality of life in end-of-life care. Honest conversations between the patient, clinicians, and the family on the disease and treatment outcomes cab serve as the platform where all the people involves in end-of-life care can come together for effective plan and policy to encounter the environmental and psychological challenges in patient terminal experiences [17].

There is no better coping mechanism than the early interventions with proper treatment plans and policy in dealing with the terminal illness diagnosis. The involvement of family loved ones, and the community inpatient terminal experience is the best coping mechanism to meet environmental demands like traveling, having quality time together, and creating good memories. The life review method is another coping mechanism that helps the patient set new life goals, which have the maximum possibilities to achieve before the inevitable death strikes. Assisting the patient in managing their financial income for treatment expenses through any possible means can serve 
as the contributing factor to improve patient quality of life and wellbeing in the clinical practices. Forming a small cell group within the cancer-affected community, including cancer survivors, to discuss their experiences, insights, feelings, in any terminal illness are other effective strategies to fight against cancer stigmas $[4,5]$. Some effective psychotherapeutic coping mechanisms are given below.

Figure 2 - Psychotherapeutic Coping Mechanism [4,5].

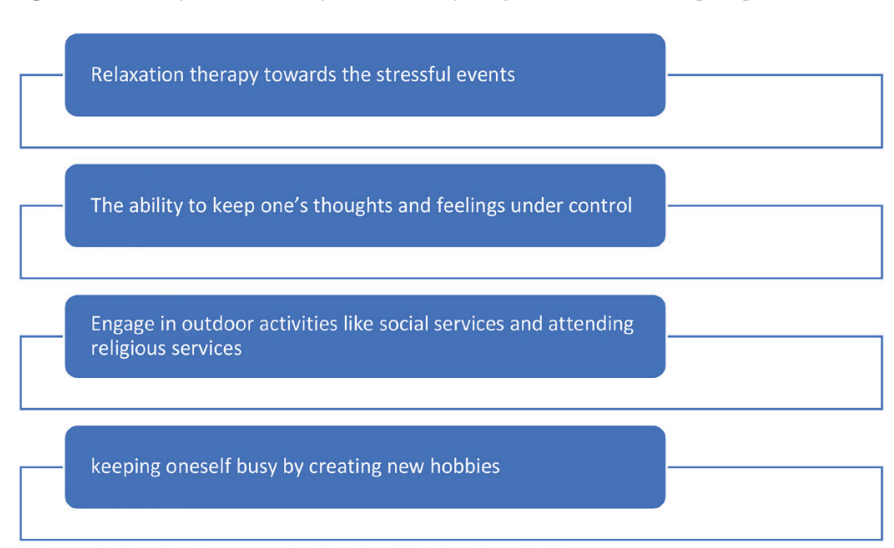

A spiritual coping mechanism is a modern humanistic approach to deal with stress and depression in the face of medical helplessness. Spiritual psychotherapy is a modern holistic approach to illness in health and medical sciences, which serves as an effective coping mechanism against several unwanted experiences like existential stress, anxiety, depression, and emotional suffering that pre-occupied the terminally ill patient in terminal diagnosis. When cure becomes uncertain in terminal diagnosis, spirituality becomes part of patient existence, potentially communion with self, others, nature, and the transcendent being. It is the coping mechanism that delivers the whole person treatment by addressing patient self-identity, inner peace, reconciliation, and hope with gratitude, which provides healing even when cure is impossible in terminal diagnosis. The involvement of psycho-social therapy is another effective coping mechanism that enables the dying individual to connect with the society where they belong. The treatment makes the patient experience the feeling of being valued by eliminating the existing social stigma of being an outcast $[18,15]$. However, the absence of anti-anxiety, anti-psychotic, and mood-stabilizing therapeutic approaches to terminal care in the current health care system in India and other neighboring countries like Nepal, Bangladesh, Pakistan, Myanmar, etc., cancer/terminal patients undergo several unwanted feelings without any quality assessment. Due to the unavailability of the whole person treatment in the country's health care system in general and in the medical curriculum, psychological stress and depression remain untouched in its clinical practices. Thus, India is considered as a country not to die by many [19].

\section{Conclusion}

The psychological stress and depression destroyed the patient peace of mind and possess the ability for cancer metastasis growth in the body parts and to disable the organs. The interplay between the environmental challenges, stressors, and the patient's socio-economic, cultural, and religious background needs special consideration in cancer diagnosis to produce the quality of life and wellbeing of the whole clinical practice. Developing positive mental health and a healthy lifestyle is the core of coping with psychological stress and depression in any terminal experience.[19,20] Moreover, even in the terminal stage when cure is not possible, finding purpose and meaning in life can benefit self and others and is also an effective coping mechanism to overcome mental health problems.

Disclosures: There is no conflict of interest for all authors.

\section{Acknowledgements: None.}

\section{Funding: None.}

Informed consent: Informed consent was obtained from all individual adult participants included in the study; assent was obtained from children.

\section{References}

1. Deimling Gary, Kahana B, Bowman FK, Schaefer Michael L. Cancer survivorship and Psychological Distress in Later Life. PsychoOncology. 2002; 11(6):479-94. DOI: 10.1002/pon.614.

2. Tan Siang Yong, Yip A. Hans Selye (1907-1982): Founder of the Stress Theory. Singapore Med J. 2018; 59(4):170-171. Doi: 10.11622/ smedj.2018043.

3. Vedprakash Sharma, Sunil Choudhary, Mona Srivastava, T. B Singh, Ravi Shankar. Prevalence of Depression, Anxiety and Stress among Cancer and Chronic Kidney Disease Patients. International Journal of Health Sciences \& Research. 2019; 9(4):1-6.

4. Debasweta Purkayastha, Chitra Venkateswaran, Kesavankutty Nayar, and UG Unnikrishnan. Prevalence of Depression in Breast Cancer Patients and its Association with their Quality of Life: A Cross-sectional Observational Study. Indian J Palliat Care. 2017; 23(3):268273. Doi: 10.4103/IJPC.IJPC_6_17.

5. Cagliostro Dina. Depression Types, Causes, Symptoms, Statistics, \& Treatment. https://www.psycom.net/depression.central.html. Accessed on December 3, 2019.

6. Encyclopedia Britannica. Depression Psychology. https://www.britannica.com/science/ depression-psychology. Accessed on December 6, 2019.

7. Annie Alexander, K Sreenath, R Srinivasa Murthy. Beyond numbers - Recent understanding of emotional needs of persons diagnosed with cancer 2007-2018. Indian Journal of Palliative care. 2020; 26(1): 120-128. https://doi.org/10.4103/IJPC.IJPC_86_19

8. Mayo Clinic. Mental Illness. http://www.mayoclinic.org/disease-conditions/mental-illness/symptoms-causes/syc-20374968. Accessed on December 4, 2019.

9. Cohen, S. Kessler, RC. Gordon UL. Strategies for Measuring Stress in Studies of Psychiatric and Physical Disorder: A Guide for Health and Social Scientists. New York: Oxford University Press, 1995. 
10. Krohne, H.W. Individual Differences in Coping. Handbook of Coping: Theory, Research, Applications. New York: Wiley Publications, 1996.

11. Myrthala Moreno-Smith, Susan K Lutgendorf \& Anil K Sood. Impact of stress on cancer metastasis. Future Oncology. 2010; 6(12):1863-1881. Doi: org/10.2217/fon. 10.142

12. McEwen BS. Protective and damaging effects of stress mediators. New England Journal of Medicine. 1998; 338(3):171-179. DOI: 10.1056/NEJM199801153380307.

13. Lloyd-William Mari. Difficulties in Diagnosing and Treating Depression in the Terminally Ill Patient. Postgrad Med Journal. 2000; 76(899):555- 558. Doi.org/10.1136/pmj. 76.899.555.

14. Reiche Edna M et al. Stress, Depression, the Immune System, and Cancer. The Lancet Oncology. 2004; 5(10):617-25. DOI: 10.1016/ S1470-2045(04)01597-9.

15. Lloyd-Williams Mari. Depression - the hidden symptom in advanced cancer. $J$ R Soc Med. 2003; 96(12):577-581. Doi: 10.1258/ jrsm.96.12.577.

16. Widera E and Block Susan D. Managing Grief and Depression at the End of Life. American Family Physician. 2012; 3(1):259-264.

17. Groninger H and Vijayan J. Pharmacologic Management of Pain at the End of Life. American Family Physician. 2014 ; 90(1):26-32.

18. Vaiphei Demkhosei suantak. The importance of holistic assessment in palliative end-of-life care and quality health outcomes. Journal of Clinical Medicine of Kazakhstan. 2019; 3(52):6-10. DOI: 10.23950/1812-2892-JCMK-00700.

19. Suantak Demkhosei Vaiphei \& Devendra Singh Sisodia. Why Cancer/Terminal Ill Diagnosis Unsuccessful in India: A Qualitative Analysis. Eurasian Journal of Medicine and Oncology-EJMO. 2019; 3(3):160-166. DOI: 10.14744/ejmo.2019.32355

20. Suantak Demkhosei Vaiphei \& Devendra Singh Sisodia. Terminal cancer in Northeast India: an analytical study on its rapid growth, causes, and solutions. The European Research Journal. 2020; 6(3):248-256. DOI: 10.18621/eurj.519115. 